\title{
Alignment enhancement of molecules embedded in helium nanodroplets by multiple laser pulses
}

\author{
Lars Christiansen and Jens H. Nielsen* \\ Department of Physics and Astronomy, Aarhus University, 8000 Aarhus C, Denmark \\ Dominik Pentlehner \\ Department of Chemistry, Aarhus University, 8000 Aarhus C, Denmark \\ Jonathan G. Underwood \\ Department of Physics and Astronomy, University College London, Gower Street, London WC1E 6BT, United Kingdom \\ Henrik Stapelfeldt \\ Department of Chemistry, Aarhus University, 8000 Aarhus C, Denmark \\ and Interdisciplinary Nanoscience Center (iNANO), Aarhus University, 8000 Aarhus C, Denmark
}

(Received 29 May 2015; published 10 November 2015)

\begin{abstract}
We show experimentally that field-free one-dimensional (1D) alignment of 1,4-diiodobenzene molecules embedded in helium nanodroplets, induced by a single, linearly polarized 200-fs laser pulse, can be significantly enhanced by using two or four optimally synchronized laser pulses. The strongest degree of $1 \mathrm{D}$ alignment is obtained with four pulses and gives $\left\langle\cos ^{2} \theta\right\rangle>0.60$. Besides the immediate implications for molecular frame studies, our results pave the way for more general manipulation of rotational motion of molecules in He droplets.
\end{abstract}

DOI: 10.1103/PhysRevA.92.053415

PACS number(s): $32.80 . \mathrm{Rm}, 42.50 . \mathrm{Hz}, 67.25 . \mathrm{dw}$

\section{INTRODUCTION}

The ability to align molecules, i.e., confine their axes with respect to axes defined in the laboratory frame, can be of great use in studies of the structure [1,2] and dynamics [3,4] of molecules. Among several methods, alignment induced by nonresonant, moderately intense laser pulses [5] has proven very versatile and efficient for isolated molecules and, as a result, found widespread applications in a variety of areas in molecular science. A particular emphasis has been on onedimensional (1D) alignment, i.e., where the most polarizable axis of a molecule is aligned along the polarization axis of a linearly polarized laser pulse. Notably, for pulses much shorter than the inherent rotational period of the molecules the alignment can exist in narrow time windows (long) after the pulse is turned off [6]. This is an attractive situation because experiments on the aligned molecules can then be conducted without any risk of perturbations from the alignment field. Unfortunately, the degree of alignment, created by a single short pulse, may not be sufficiently high that the complete molecular frame information or the detailed orientational dependence of molecular processes is fully elucidated.

To improve the degree of 1D alignment-and maintain field-free conditions-a sequence of short laser pulses, synchronized such that each pulse is sent at the time when the degree of alignment induced by the previous pulse in the sequence peaks, can be used. This idea was introduced and analyzed in Ref. [7] and the first experiments demonstrating the effect on isolated molecules, employing two alignment pulses, were reported shortly after $[8,9]$. Since then, many more works, theoretically as well as experimentally, have further explored

\footnotetext{
*Present address: Research Software Development Group, Research IT Services, University College London, Podium Building (1st Floor), Gower Street, London WC1E 6BT, United Kingdom.
}

the use of laser pulse sequences and shaped laser pulses to optimize and control rotations of isolated molecules, including demonstration of eight-pulse alignment [10], field-free 3D alignment [11,12], permanent planar alignment [13], and controlling the sense of molecular rotation [14-17].

In the current work we show experimentally that alignment enhancement by a sequence of synchronized short laser pulses also applies to molecules embedded in helium nanodroplets. The motivation for our work is twofold. First, alignment of molecules inside He droplets, only reported for the first time in 2013 [18,19], opens novel possibilities for studying molecular frame reaction dynamics in the presence of a dissipative environment. This ultimately requires sharply aligned molecules, preferably under field-free conditions. The results reported here are a step in that direction. Second, the ability to enhance the degree of alignment by pulse trains implies being able to control the rotational angular momentum and coherence of the molecules inside the droplet. Since some or all of the angular momentum and coherence may be transferred to the surrounding $\mathrm{He}$ atoms, our work points to possibilities for laser-controlled excitation of collective angular momentum states, possibly quantized vortices [20-22] if more extreme laser pulse forms are employed.

\section{EXPERIMENTAL SETUP}

Most of the experimental setup has been described previously $[18,19]$ and only the important features are discussed here. The He droplets are formed by continuously expanding $\sim 25$ bar $\mathrm{He}$ gas, cryogenically precooled to $12 \mathrm{~K}$, through a $5 \mu \mathrm{m}$ orifice into vacuum. At this temperature the droplets consist on average of $\sim 1.5 \times 10^{4} \mathrm{He}$ atoms [23]. The droplet beam passes through a pick-up cell, where the partial pressure of 1,4 diiodobenzene (DIB), $\mathrm{C}_{6} \mathrm{H}_{4} \mathrm{I}_{2}$ [structure shown in Fig. 1(a)], is adjusted to optimize for single-molecule doping of each droplet. Hereafter, the doped He droplet beam enters the target 

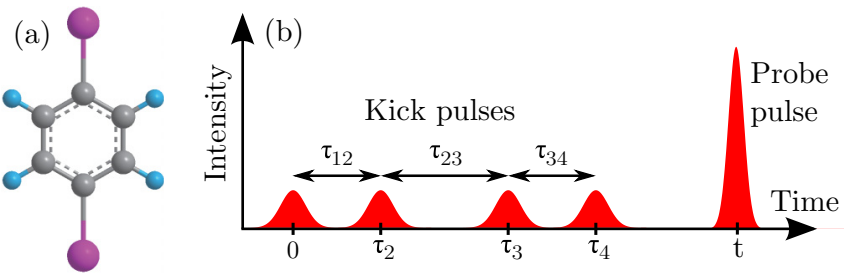

FIG. 1. (Color online) (a) A model of the DIB molecule. (b) Illustration of the laser pulse timings. The center of the first kick pulse defines the zero of time. A two-pulse sequence of the kick pulses is denoted $\left(0-\tau_{2}\right)$ and a four-pulse sequence $\left(0-\tau_{2}-\tau_{3}-\tau_{4}\right)$.

region where it is crossed, at $90^{\circ}$, by two collinear, focused, pulsed laser beams. The pulses in the first beam (kick pulses: $\lambda=800 \mathrm{~nm}, \tau_{\mathrm{FWHM}}=200 \mathrm{fs}, \mathrm{I}_{\text {kick }}=1.6 \times 10^{13} \mathrm{Wcm}^{-2}$ ) are linearly polarized and serve to induce $1 \mathrm{D}$ alignment. This implies alignment of the I-I axis since this is the most polarizable axis of the molecule. The four-pulse sequence is obtained by passing part of the output beam from an amplified Ti:Sa system through a pair of Mach-Zehnder-type setups. This creates two pairs of laser pulses where the time separation between pulse 1 and $2, \tau_{12}$, and between pulse 3 and $4, \tau_{34}$, is identical but can be chosen freely, as can the time separation between pulse 2 and $3, \tau_{23}$, see Fig. 1(b). The two-pulse sequence is obtained by using only the first Mach-Zehnder setup. The pulses in the second beam (probe pulse: $\lambda=$ $800 \mathrm{~nm}, \tau_{\text {FWHM }}=30 \mathrm{fs}, \mathrm{I}_{\text {probe }}=3.8 \times 10^{14} \mathrm{Wcm}^{-2}$ ) are used to Coulomb explode the molecules. This enables determination of the degree of alignment when the emission direction of the $\mathrm{I}^{+}$fragments are recorded-here achieved by a velocity map imaging spectrometer. Assuming axial recoil in the explosion process, the recoil direction of the $\mathrm{I}^{+}$ions is a direct measure of the molecular I-I axis distribution at the time, $t$, of the probe pulse [24] defined with respect to the first kick pulse [Fig. 1(b)]. As such, the main observables are two-dimensional $\mathrm{I}^{+}$ion images. To enable comparison of alignment of molecules in $\mathrm{He}$ droplets and of isolated molecules under identical laser pulse conditions, a second molecular beam, consisting of isolated DIB molecules, can be sent into the target region.

\section{EXPERIMENTAL RESULTS}

The upper row of Fig. 2 shows examples of $\mathrm{I}^{+}$ion images from ionization of DIB molecules in He droplets. When only the probe pulse, linearly polarized perpendicular to the detector plane, is used the $\mathrm{I}^{+}$image is circularly symmetric as expected for randomly oriented molecules [Fig. 2(a1)]. When a single kick pulse is included the $\mathrm{I}^{+}$ions localize along the kick pulse polarization at certain times. At these times the I-I axis of the molecule is aligned. An image, recorded at $t=40 \mathrm{ps,}$ is displayed in Fig. 2(a2). To quantify how the degree of alignment varies with time $\left\langle\cos ^{2} \theta_{2 \mathrm{D}}\right\rangle$ is determined for each image, recorded at different delays. Here $\theta_{2 \mathrm{D}}$ is the angle between the kick pulse polarization and the projection of the $\mathrm{I}^{+}$ ion recoil velocity vector on the detector screen. The $\left\langle\cos ^{2} \theta_{2 \mathrm{D}}\right\rangle$ values are determined from the ions produced due to Coulomb explosion of the molecules, which are the ions in the region between the white circles shown in Fig. 2.

Figure 3(a1) displays the time dependence of $\left\langle\cos ^{2} \theta_{2 \mathrm{D}}\right\rangle$ obtained with a single kick pulse. The graph shows that

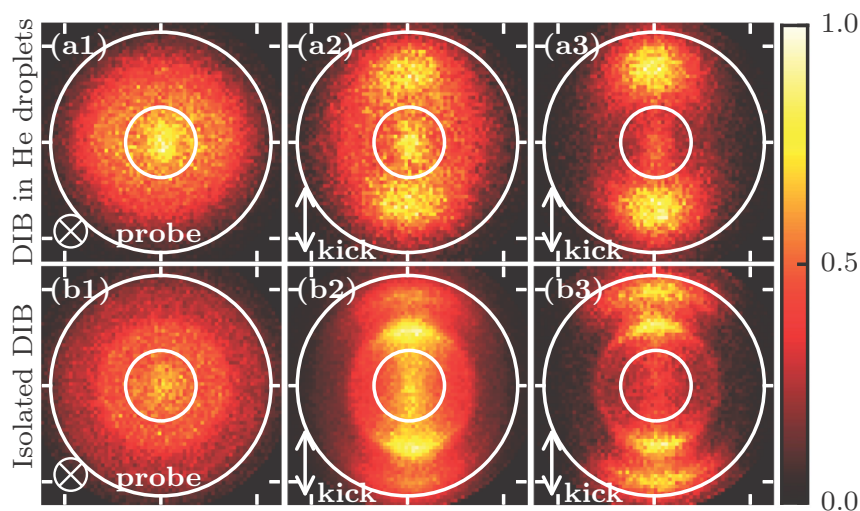

FIG. 2. (Color online) $\mathrm{I}^{+}$ion images from DIB molecules in $\mathrm{He}$ droplets [(a1)-(a3)] and from isolated DIB molecules [(b1)-(b3)]. Each image is $37 \mathrm{~mm}$ by $37 \mathrm{~mm}$ and each tick separation is $15 \mathrm{~mm}$. Column (1): probe pulse only. Column (2): Strongest alignment with one kick pulse; $t=40 \mathrm{ps}$ in (a2) and $t=12 \mathrm{ps}$ in (b2). Column (3): Strongest alignment with four kick pulses. In (a3) the pulse sequence is (0-18-36-54) ps and $t=70 \mathrm{ps}$; in (b3) the pulse sequence is (0-2$8-10) \mathrm{ps}$ and $t=14 \mathrm{ps}$. The probe pulse is polarized perpendicular to and the kick pulses parallel to the image plane, indicated in each panel.

$\left\langle\cos ^{2} \theta_{2 \mathrm{D}}\right\rangle$ rises monotonically from $\sim 0.50$, the value characterizing randomly oriented molecules, just before the kick pulse to a maximum of 0.60 at $t=40 \mathrm{ps}$. Hereafter, $\left\langle\cos ^{2} \theta_{2 \mathrm{D}}\right\rangle$ decreases steadily and returns to $\sim 0.50$ around $t=150 \mathrm{ps}$.

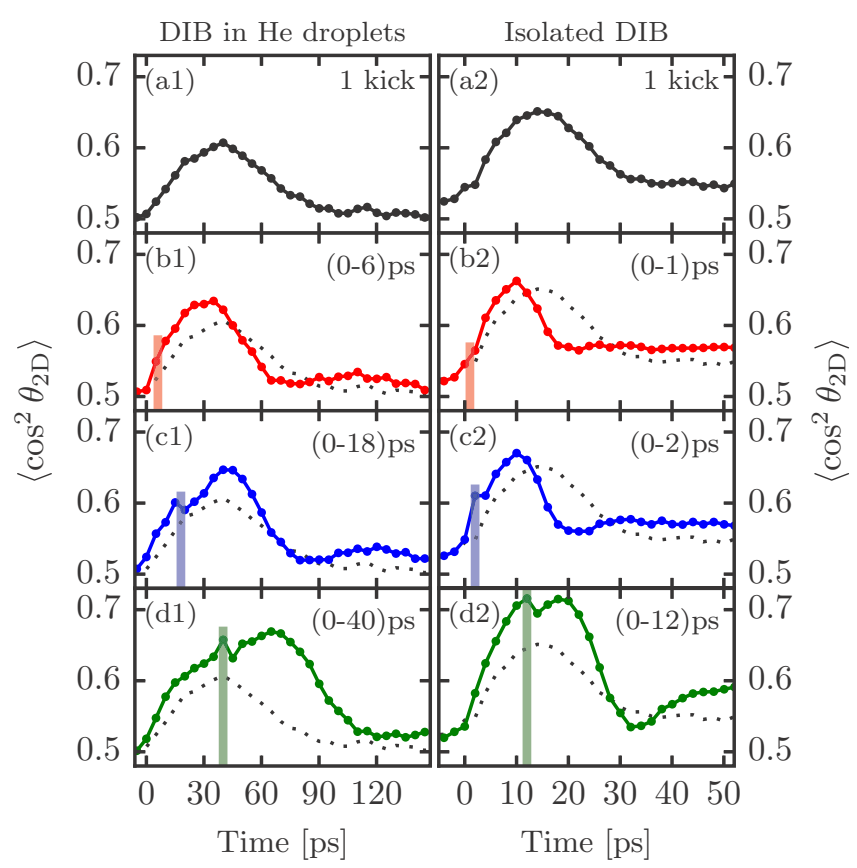

FIG. 3. (Color online) The degree of alignment, represented by $\left\langle\cos ^{2} \theta_{2 \mathrm{D}}\right\rangle$, as a function of time for DIB in He droplets, column (1) and isolated DIB molecules, column (2). Row (a): Result for a single kick pulse, row (b)-(d): Results for different two-pulse sequences with the one-pulse results retained as a reference (dotted). $\left\langle\cos ^{2} \theta_{2 \mathrm{D}}\right\rangle$ is only correctly representing the degree of alignment after the second kick pulse (marked by the vertical bars), see text. The pulse sequence is given in the top right of each panel. 
This alignment dynamic is very similar to that recently observed for $\mathrm{C}_{6} \mathrm{H}_{5} \mathrm{I}$ and $\mathrm{CH}_{3} \mathrm{I}$ molecules in He droplets [18]. It is not possible to increase $\left\langle\cos ^{2} \theta_{2 \mathrm{D}}\right\rangle$ further by increasing $\mathrm{I}_{\mathrm{kick}}$ because its value $\left(1.6 \times 13 \mathrm{Wcm}^{-2}\right)$ was set to the maximum the molecules could withstand without undergoing significant ionization (and fragmentation). In the two-pulse and four-pulse measurements discussed below $\mathrm{I}_{\text {kick }}$ of each pulse is kept at this value, which ensured negligible ionization of the molecules by any of the pulse sequences used.

Next, alignment with two identical kick pulses was investigated. Initially, the second pulse was sent at the time $(t=40 \mathrm{ps})$ when the alignment induced by the first pulse peaks - motivated by studies on isolated molecules $[8,9,25]$. The result, displayed in Fig. 3(d1), shows that after the second pulse $\left\langle\cos ^{2} \theta_{2 \mathrm{D}}\right\rangle$ rises to a new maximum of 0.67 at $t \sim 70 \mathrm{ps}$. This demonstrates that the two-pulse alignment enhancement method, established for gas phase molecules $[8,9,25]$ is transferable to molecules inside $\mathrm{He}$ droplets. Other kickpulse separations were investigated and the results for time separation of 6 ps and 18 ps are shown in Figs. 3(b1) and 3(c1), respectively. In both cases an enhanced degree of alignment is achieved after pulse number two ( 0.63 and 0.65 , respectively) but not as pronounced as for the case in Fig. 3(d1). If the time between the kick pulses is increased beyond 40 ps the maximum degree of alignment also decreases. These findings follow the trend observed for two-pulse alignment of isolated molecules [8].

We note that in each of Figs. 3(b1), 3(c1), and 3(d1), the $\left\langle\cos ^{2} \theta_{2 \mathrm{D}}\right\rangle$ curve recorded with two kick pulses lies higher than the $\left\langle\cos ^{2} \theta_{2 \mathrm{D}}\right\rangle$ curve recorded with one kick pulse (black) for times before the second pulse is sent although the alignment ought to be identical since pulse number two has not arrived yet. We believe this is due to the second kick pulse fragmenting or further ionizing some (metastable) molecular ions, formed by the probe pulse. This fragmentation or ionization process is favored for molecules aligned along the kick pulse polarization [26]. As such, the $\left\langle\cos ^{2} \theta_{2 \mathrm{D}}\right\rangle$ values for the two-pulse sequence are not faithfully representing the degree of alignment until all kick pulses have been sent. In particular, this implies that $\left\langle\cos ^{2} \theta_{2 \mathrm{D}}\right\rangle$ measured before $t=0$ is also biased, even when only a single kick pulse is used. This explains why $\left\langle\cos ^{2} \theta_{2 \mathrm{D}}\right\rangle$, for $t<0$, is slightly larger than 0.50 for all curves in Fig. 3. Similar considerations hold for the four-pulse data (Fig. 4) discussed next.

Finally, alignment induced by four kick pulses was studied and examples of the rotational dynamics recorded are presented in Fig. 4. In Fig. 4(a1) the pulse sequence (0-6-16-22) ps was used. The maximum of $\left\langle\cos ^{2} \theta_{2 \mathrm{D}}\right\rangle, 0.69$, is reached at $36 \mathrm{ps}$, i.e., $14 \mathrm{ps}$ after the fourth pulse. This value already represents an improvement over the strongest two-pulse alignment. If $\tau_{23}$ is increased to $18 \mathrm{ps}$ the maximum value of $\left\langle\cos ^{2} \theta_{2 \mathrm{D}}\right\rangle$ increases slightly to 0.70 at 45 ps [Fig. 4(b1)]. We believe the improvement occurs because the time-dependent degree of alignment induced by the first pulse pair reaches a higher value after $18 \mathrm{ps}$ compared to after $10 \mathrm{ps}$. This is advantageous for the degree of alignment the second pulse pair can induce - see for instance $[7,27,28]$ and the discussion in Sec. IV. Keeping $\tau_{12}=\tau_{34}$ fixed at 6 ps and increasing $\tau_{23}$ further we observe slightly lower maximum $\left\langle\cos ^{2} \theta_{2 \mathrm{D}}\right\rangle$ values, for instance for the sequence (0-6-31-37) ps $\left\langle\cos ^{2} \theta_{2 \mathrm{D}}\right\rangle$ reaches 0.69 at 55 ps.

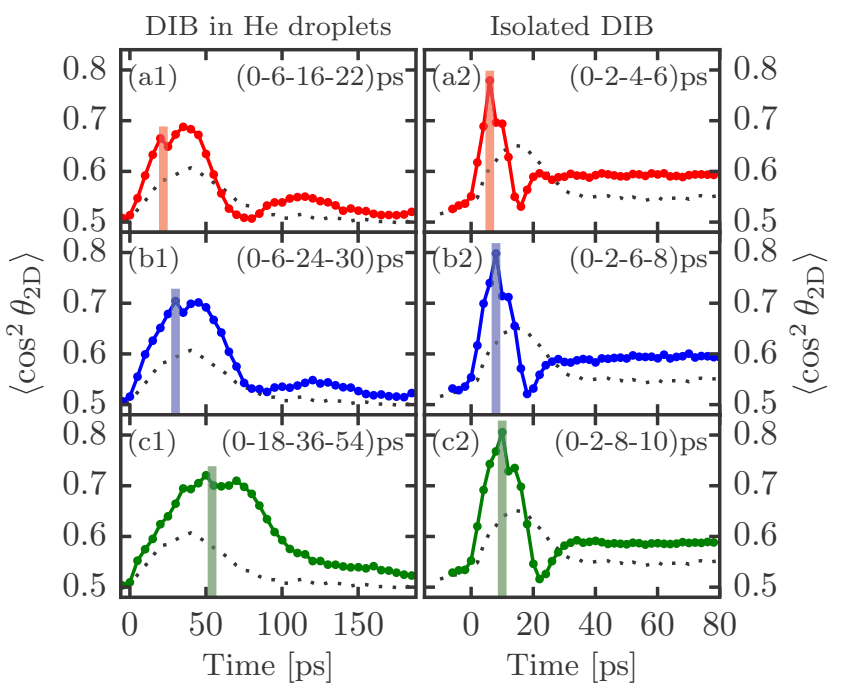

FIG. 4. (Color online) The degree of alignment, represented by $\left\langle\cos ^{2} \theta_{2 \mathrm{D}}\right\rangle$, as a function of time when four kick pulses are used to induce alignment. Column (1): DIB in He droplets. Column (2): Isolated DIB molecules. Row (a)-(c) show the results for three different four-pulse sequences. In each panel the result obtained with a single kick pulse is displayed as a reference (dotted). $\left\langle\cos ^{2} \theta_{2 \mathrm{D}}\right\rangle$ is only correctly representing the degree of alignment after the last kick pulse (marked by the vertical bars), see text. The pulse sequence is given in the top right of each panel.

Other pulse sequences were explored with larger values of $\tau_{12}=\tau_{34}$ and for different $\tau_{23}$ values. Within the limited parameter space we explored, the (0-18-36-54) ps sequence [Fig. 4(c1)] yielded the strongest alignment, $\left\langle\cos ^{2} \theta_{2 \mathrm{D}}\right\rangle=$ 0.71 , at $t=70 \mathrm{ps}$. The corresponding $\mathrm{I}^{+}$ion image is displayed in Fig. 2(a3). Our results demonstrate that a four-pulse sequence can enhance the degree of alignment beyond that achievable with a two-pulse sequence although a systematic search for the pulse sequence that optimizes $\left\langle\cos ^{2} \theta_{2 \mathrm{D}}\right\rangle$ was not possible due to the restrictions $\left(\tau_{12}=\tau_{34}\right)$, imposed by the optical setup. If $\tau_{12}, \tau_{23}, \tau_{34}$ could be chosen freely, for instance by employing a pulse shaper, we anticipate a (significant) enhancement of $\left\langle\cos ^{2} \theta_{2 \mathrm{D}}\right\rangle$, in particular for a pulse sequence where each pulse is sent when the degree of alignment from the previous pulses peaks. The results of Fig. 3(d1) identify the sequence of the first three pulses to (0-40-70) ps and likely the fourth should follow 10-20 ps after the third pulse.

For comparison we also recorded the alignment dynamics of isolated DIB molecules for intensities and durations of the kick and probe pulses identical to those used in the droplet studies. The $\left\langle\cos ^{2} \theta_{2 \mathrm{D}}\right\rangle$ curve recorded with a single kick pulse [Fig. 3(a2)] reaches a maximum (0.65) at $t \sim 14$ ps. This is about 2.8 times faster than for the molecules in the He droplets and consistent with previous observations [18]. The subsequent decrease of $\left\langle\cos ^{2} \theta_{2 \mathrm{D}}\right\rangle$ ends at $\sim 0.55$ rather than at 0.50 observed for the droplet case. This alignment is often termed permanent alignment. It is a manifestation of the coherent superposition of rotational eigenstates, created by the kick pulse in each DIB molecule, being preserved at long times. The coherence leads to rotational revivals, for instance, we observed a distinct revival centered around 1580 ps [29]. No revivals are observed for the droplet case [18]. 
Results for two-pulse alignment at some of the kick pulse separation times recorded are shown in Figs. 3(b2)-3(d2). Similar to previous studies on isolated molecules the strongest alignment occurs when the second pulse arrives around the time where $\left\langle\cos ^{2} \theta_{2 \mathrm{D}}\right\rangle$ induced by the first pulse peaks. In particular, Fig. 3(d2) shows that $\left\langle\cos ^{2} \theta_{2 \mathrm{D}}\right\rangle$ reaches 0.71 at $t=18 \mathrm{ps}$ when the second kick pulse is sent at $t=12 \mathrm{ps}$. With four kick pulses the highest $\left\langle\cos ^{2} \theta_{2 D}\right\rangle$ value was found for the pulse sequence (0-2-8-10) ps, see Fig. 4(c2). Here $\left\langle\cos ^{2} \theta_{2 \mathrm{D}}\right\rangle$ rises to a peak value of 0.74 at $t=14$ ps demonstrating that the degree of field-free alignment can be enhanced by using four pulses compared to two pulses. The $\mathrm{I}^{+}$ion image corresponding to the best four-pulse alignment is displayed in Fig. 2(b3) along with ion images obtained with a single kick pulse [Fig. 2(b2)] and the probe pulse only [Fig. 2(b1)]. As for the droplet measurements $\left\langle\cos ^{2} \theta_{2 \mathrm{D}}\right\rangle$ is only correctly describing the alignment after all kick pulses are sent.

We note that $\left\langle\cos ^{2} \theta_{2 D}\right\rangle$ gives only a relative measure for the degree of alignment primarily because the efficiency of probing a given molecule depends on how it is oriented in space. The effect results from the dependence of strong field ionization on molecular alignment [30] and implies that the best aligned molecules are probed least efficiently because the probe pulse polarization is perpendicular to the polarization of the kick pulses. To compensate for the nonuniform probe efficiency we applied a recently reported method [31], which retrieves the true I-I axis distribution of the $1 \mathrm{D}$ aligned DIB molecules. From this distribution $\left\langle\cos ^{2} \theta\right\rangle$ is determined, where $\theta$ is the polar angle between the $\mathrm{I}^{+}$emission angle and the polarization axis of the kick pulses. The method is implemented for DIB in He droplets and shows that $\left\langle\cos ^{2} \theta\right\rangle$ increases from $\sim 0.47$ for one laser pulse to $\sim 0.60$ for the best four-pulse sequence.

The measurements are also influenced by the deviation from axial recoil in the Coulomb explosion process. Covariance analysis of the $\mathrm{I}^{+}$angular distribution shows that the emission direction of the $\mathrm{I}^{+}$ions deviates with up to $\pm 15^{\circ}$ from the parent I-I axis for isolated molecules and up to $\pm 20^{\circ}$ for molecules in He droplets. This implies that the $\left\langle\cos ^{2} \theta\right\rangle$ values quoted above underestimate the true degree of alignment.

\section{DISCUSSION}

In addition to demonstrating enhanced alignment the $\left\langle\cos ^{2} \theta_{2 \mathrm{D}}\right\rangle$ traces recorded also provide information about the rotational wave packets formed. This is discussed in this section. For isolated DIB molecules the right column in Fig. 3 shows that the rotational dynamics is faster when two rather than one kick pulse is used. In particular, the peak alignment occurs about 14 ps after the first pulse whereas the time for reaching peak alignment after the second kick pulse is reduced to $9 \mathrm{ps}, 8 \mathrm{ps}$, and $7 \mathrm{ps}$ for the two-pulse sequences [(0-1) ps, (0-2) ps, and (0-12) ps] depicted in Fig. 3. In addition, the decay of the peak alignment occurs faster for the two-pulse sequences (about 10 ps) than for the single kick pulse (about $20 \mathrm{ps}$ ).

These observations can be rationalized both classically and quantum mechanically. In a classical model $[7,27,28]$ molecules initially aligned at a polar angle $\theta_{0}$ with respect to the laser polarization acquire an angular velocity towards the laser polarization of $\omega\left(\theta_{0}\right) \propto \sin 2 \theta_{0}$ after the pulse if zero initial angular velocity is assumed. When the second kick pulse is sent while the molecules rotate towards the kick pulse polarization the angular speed will increase and peak alignment is reached faster. In addition, the increased angular speed results in a more rapid loss of alignment, which is what we observe.

The classical model also offers an explanation for the enhanced degree of alignment and the optimal timing of the kick pulses. After the application of a single pulse all molecules rotate with an angular speed $\propto \sin 2 \theta_{0}$. Therefore, molecules starting at small angles $\left(\sin 2 \theta_{0} \approx 2 \theta_{0}\right)$ rotate into alignment with the polarization axis at approximately the same time, whereas rotors starting at large angles lag behind, independent of the kick strength. The difference in time at which alignment is reached for different initial orientations $\left(\theta_{0}\right)$ implies a limited overall degree of alignment for the sample. The limitation can be reduced if a prealignment pulse lowers the average polar angle prior to the kick of a second alignment pulse. This average polar angle is minimized when the degree of alignment induced by the first pulse peaks and thus explains why the second pulse should be sent around this time, in line with the experimental findings. This timing strategy was termed accumulative squeezing and also applies to alignment induced by more than two laser pulses [28].

Quantum mechanically, the two-pulse sequence will enhance the average angular momentum, $\sqrt{\left\langle\mathbf{J}^{2}\right\rangle}$, of the molecule [10] and so broaden the $J$-state composition of the rotational wave packet similar to the effect that happens when the intensity of a single alignment pulse is increased [32]. The larger width of the wave packet in $J$ space, i.e., the larger $\left\langle\mathbf{J}^{2}\right\rangle$, can result in enhanced alignment when the timing of the second pulse is correct. A higher $\left\langle\mathbf{J}^{2}\right\rangle$ also leads to both faster initial dynamics, i.e., an earlier occurrence of the alignment peak after the final kick pulse and faster dispersion, i.e., faster decay of the peak alignment maximum. In addition, as well as enhancing $\left\langle\mathbf{J}^{2}\right\rangle$, our analysis of the molecular axis distribution with the nonuniform probe efficiency taken into account [31] shows that a greater degree of $J$-state coherence is produced for the four-pulse experiment.

When four kick pulses (right column of Fig. 4) are used the rotational dynamics following the fourth pulse is even faster than the dynamics for the two-pulse case. This points to a further increase of the width in $J$ space of the rotational wave packet in line with the expectations when four rather than two pulses are used [10]. In addition, Fig. 3 shows that the permanent alignment level increases from 0.55 when a single pulse is used [Fig. 3(a2)] to 0.57 for the $(0-1)$ ps and (0-2) ps sequences [Figs. 3(b2) and 3(c2)] and to 0.58 for the (0-12) ps sequence [Fig. 3(d2)] and, finally, to 0.60 when four pulses are used (right column of Fig. 4). These observations are consistent with a shift towards higher $J$ states of the rotational wave packet as the number of kick pulses is increased.

Turning next to the data for molecules in He droplets we note that here the rotational dynamics is also faster when two kick pulses rather than one is used as can be seen in the left column of Fig. 3. Similar to the isolated molecule case this observation can be interpreted as the rotational wave packet created contains higher $J$ states when two kick pulses are used. The data recorded with four kick pulses are less unambiguous. For the (0-6-16-22) ps and the 0-6-24-30) ps 
sequences the rotational dynamics appear faster than for any of the two-pulse sequences whereas the dynamics induced by the (0-18-36-54) ps sequence is significantly slower than for the other four-pulse sequences. The (0-18-36-54) ps sequence is the one that extends over the largest time, i.e., the time separation between the pulses is the largest, and it is possible that loss of coherence of the wave packet or population decay of its rotational state components, mediated through interaction with the surrounding He environment [33], is significant on this time scale, which would reduce the final coherence of the wave packet after all four pulses. An indication that this may indeed be the case is the fact that the oscillation in the $\left\langle\cos ^{2} \theta_{2 \mathrm{D}}\right\rangle$ trace observed for the (0-6-16-22) ps and the (0-6-24-30) ps are absent for the 0-18-36-54) ps sequence. These oscillations are a manifestation of the coherences between the rotational states in the wave packet and they are pronounced in the isolated molecule $\left\langle\cos ^{2} \theta_{2 \mathrm{D}}\right\rangle$ traces, both for the two- and-four-pulse sequences. For molecules in He droplets the oscillations seem most pronounced for the four-pulse sequence with the smallest pulse separation, (0-6-16-22) ps, where the molecules have had the least amount of time to lose their coherence due to the interaction with the droplet. The current data are insufficient to draw definite conclusions about the time-dependent alignment dynamics of molecules in He droplets but it appears that studies of the rotational dynamics induced by sequences of kick pulses where the pulse separation is systematically varied from very short to long times have a potential for shedding new light on the interaction between rotating molecules and the surrounding He environment.

\section{CONCLUSION}

In conclusion we have shown that properly synchronized sequences of two- or four-femtosecond laser pulses can significantly enhance the degree of 1D field-free alignment of molecules inside He droplets compared to that achievable with a single laser pulse. The findings were based on studies of DIB molecules but the technique should apply to a range of molecular species. As an illustration we conducted studies on $\mathrm{CH}_{3} \mathrm{I}$ and $\mathrm{CS}_{2}$ molecules in He droplets and showed that the highest $\left\langle\cos ^{2} \theta_{2 \mathrm{D}}\right\rangle$ value increases from 0.62 to 0.67 for $\mathrm{CH}_{3} \mathrm{I}$ and from 0.58 to 0.63 for $\mathrm{CS}_{2}$ when two rather than one pulse is used to induce alignment. We emphasize that an optical setup producing multiple pulses with free tunability of their time separations is expected to enhance the alignment compared to the results reported here.

The current findings will be useful for studies or exploitation of the orientational dependence of molecular interactions as well as for extraction of molecular frame information. They may also provide more insight into development of the coherence and state populations of rotational wave packets created in molecules inside helium droplets. In addition, our work paves the way for using laser-controlled rotation of molecules to transfer large amounts of angular momenta and coherence to the helium droplet. An intriguing direction is to apply an optical centrifuge [34-37] to accelerate the rotation of a molecule, thereby stirring the droplet from within, as a means to possibly control the formation of quantum vortices [20-22].

\section{ACKNOWLEDGMENTS}

H.S. acknowledges European Research Council-AdG (Project No. 320459, DropletControl), Lundbeckfonden, Carlsbergfondet, and Natur og Univers, Det Frie Forskningsråd.

L.C. and D.P. contributed equally to this work.
[1] C. J. Hensley, J. Yang, and M. Centurion, Phys. Rev. Lett. 109, 133202 (2012).

[2] J. Küpper et al., Phys. Rev. Lett. 112, 083002 (2014).

[3] C. Z. Bisgaard, O. J. Clarkin, G. Wu, A. M. D. Lee, O. Gessner, C. C. Hayden, and A. Stolow, Science 323, 1464 (2009).

[4] J. L. Hansen, J. H. Nielsen, C. B. Madsen, A. T. Lindhardt, M. P. Johansson, T. Skrydstrup, L. B. Madsen, and H. Stapelfeldt, J. Chem. Phys. 136, 204310 (2012).

[5] H. Stapelfeldt and T. Seideman, Rev. Mod. Phys. 75, 543 (2003).

[6] F. Rosca-Pruna and M. J. J. Vrakking, Phys. Rev. Lett. 87, 153902 (2001).

[7] M. Leibscher, I. S. Averbukh, and H. Rabitz, Phys. Rev. Lett. 90, 213001 (2003).

[8] C. Z. Bisgaard, M. D. Poulsen, E. Péronne, S. S. Viftrup, and H. Stapelfeldt, Phys. Rev. Lett. 92, 173004 (2004).

[9] K. F. Lee, I. V. Litvinyuk, P. W. Dooley, M. Spanner, D. M. Villeneuve, and P. B. Corkum, J. Phys. B 37, L43 (2004).

[10] J. P. Cryan, P. H. Bucksbaum, and R. N. Coffee, Phys. Rev. A 80, 063412 (2009).

[11] K. F. Lee, D. M. Villeneuve, P. B. Corkum, A. Stolow, and J. G. Underwood, Phys. Rev. Lett. 97, 173001 (2006).

[12] X. Ren, V. Makhija, and V. Kumarappan, Phys. Rev. Lett. 112, 173602 (2014).
[13] M. Z. Hoque, M. Lapert, E. Hertz, F. Billard, D. Sugny, B. Lavorel, and O. Faucher, Phys. Rev. A 84, 013409 (2011).

[14] S. Fleischer, Y. Khodorkovsky, Y. Prior, and I. S. Averbukh, New J. Phys. 11, 105039 (2009).

[15] K. Kitano, H. Hasegawa, and Y. Ohshima, Phys. Rev. Lett. 103, 223002 (2009).

[16] S. Zhdanovich, A. A. Milner, C. Bloomquist, J. Floß, I. S. Averbukh, J. W. Hepburn, and V. Milner, Phys. Rev. Lett. 107, 243004 (2011).

[17] G. Karras, M. Ndong, E. Hertz, D. Sugny, F. Billard, B. Lavorel, and O. Faucher, Phys. Rev. Lett. 114, 103001 (2015).

[18] D. Pentlehner, J. H. Nielsen, A. Slenczka, K. Mølmer, and H. Stapelfeldt, Phys. Rev. Lett. 110, 093002 (2013).

[19] D. Pentlehner, J. H. Nielsen, L. Christiansen, A. Slenczka, and H. Stapelfeldt, Phys. Rev. A 87, 063401 (2013).

[20] F. Stienkemeier and K. K. Lehmann, J. Phys. B 39, R127 (2006).

[21] L. F. Gomez, E. Loginov, and A. F. Vilesov, Phys. Rev. Lett. 108, 155302 (2012).

[22] L. F. Gomez et al., Science 345, 906 (2014).

[23] J. P. Toennies and A. F. Vilesov, Angew. Chem., Int. Ed. 43, 2622 (2004). 
[24] J. J. Larsen, H. Sakai, C. P. Safvan, I. Wendt-Larsen, and H. Stapelfeldt, J. Chem. Phys. 111, 7774 (1999).

[25] C. Z. Bisgaard, S. S. Viftrup, and H. Stapelfeldt, Phys. Rev. A 73, 053410 (2006).

[26] E. Constant, H. Stapelfeldt, and P. B. Corkum, Phys. Rev. Lett. 76, 4140 (1996).

[27] I. S. Averbukh and R. Arvieu, Phys. Rev. Lett. 87, 163601 (2001).

[28] M. Leibscher, I. S. Averbukh, and H. Rabitz, Phys. Rev. A 69, 013402 (2004).

[29] M. D. Poulsen, E. Péronne, H. Stapelfeldt, C. Z. Bisgaard, S. S. Viftrup, E. Hamilton, and T. Seideman, J. Chem. Phys. 121, 783 (2004).

[30] J. L. Hansen, L. Holmegaard, J. H. Nielsen, H. Stapelfeldt, D. Dimitrovski, and L. B. Madsen, J. Phys. B 45, 015101 (2012).
[31] J. G. Underwood, I. Procino, L. Christiansen, J. Maurer, and H. Stapelfeldt, Rev. Sci. Instrum. 86, 073101 (2015).

[32] E. Péronne, M. D. Poulsen, C. Z. Bisgaard, H. Stapelfeldt, and T. Seideman, Phys. Rev. Lett. 91, 043003 (2003).

[33] M. Y. Choi, G. E. Douberly, T. M. Falconer, W. K. Lewis, C. M. Lindsay, J. M. Merritt, P. L. Stiles, and R. E. Miller, Int. Rev. Phys. Chem. 25, 15 (2006).

[34] D. M. Villeneuve, S. A. Aseyev, P. Dietrich, M. Spanner, M. Y. Ivanov, and P. B. Corkum, Phys. Rev. Lett. 85, 542 (2000).

[35] L. Yuan, S. W. Teitelbaum, A. Robinson, and A. S. Mullin, Proc. Natl. Acad. Sci. USA 108, 6872 (2011).

[36] A. Korobenko, A. A. Milner, J. W. Hepburn, and V. Milner, Phys. Chem. Chem. Phys. 16, 4071 (2014).

[37] A. Korobenko, A. A. Milner, and V. Milner, Phys. Rev. Lett. 112, 113004 (2014). 